\title{
COMMUNICATION
}

Cite this: DOI: $10.1039 /$ xoxxooooox

Received ooth January 2012,

Accepted ooth January 2012

DOI: 10.1039/xoxxooooox

www.rsc.org/

\section{Potassium-Alkyl Magnesiates: Synthesis, Structures and Mg-H Exchange Applications of Aromatic and Heterocyclic Substrates}

\author{
Sharon E. Baillie, ${ }^{a}$ Tobias Bluemke, ${ }^{a}$ William Clegg ${ }^{b}$ A. R. Kennedy ${ }^{a}$ Jan Klett, ${ }^{a}$ \\ Luca Russo, ${ }^{b}$ Marco de Tullio ${ }^{a}$ and Eva Hevia* ${ }^{a}$
}

Using structurally well-defined dipotassiumtetra(alkyl)magnesiates, a new straightforward methodology to promote regioselective $\mathrm{Mg}-\mathrm{H}$ exchange reactions of a wide range of aromatic and heteroaromatic substrates is disclosed. Contacted ion pair intermediates are likely to be involved, with $\mathrm{K}$ being the key to facilitate the magnesiation processes.

Metallation, where an unreactive $\mathrm{C}-\mathrm{H}$ bond is regioselectively transformed into a more reactive $\mathrm{M}-\mathrm{C}$ bond, is a popular synthetic tool for functionalizing aromatic molecules. ${ }^{1}$ Organolithium reagents are extensively employed for this purpose; however their usage usually offer limited functional group tolerance and generate lowstability intermediates, often imposing sub-ambient temperatures protocols. ${ }^{1}$ A developing alternative strategy is to use alkali-metal magnesiates. ${ }^{2}$ Operating by cooperative effects, these bimetallic reagents often display enhanced metallating power in direct (onestep) magnesiations of aromatic substrates containing a rich variety of functional groups often incompatible with organolithium reagents. Notable new reagents to this field are Knochel's "turbo-charged" reagent (TMP) MgX.LiCl ( $\mathrm{TMP}=2,2,6,6$-tetramethylpiperidide), and $\mathrm{Na}-\mathrm{Mg}$ TMP-alkyl mixtures in alkali-metal-mediated magnesiation (AMM $M g$ ) processes which can facilitate remote deprotonation of organic substrates. $^{3}$

Interestingly most studies have focused on lithium or sodium magnesiates, with little attention paid to their potassium congeners, ${ }^{4}$ reflecting the greater challenge of manipulating and isolating their potassium alkyl precursors. ${ }^{5}$ Common in existing magnesiate reagents, the amide TMP appears to be the kinetic key driving their $\mathrm{Mg}-\mathrm{H}$ exchange processes. Notwithstanding in some cases, concomitantly released TMP $(\mathrm{H})$ can react with the some residual anionic groups of the generated organometallic intermediate. ${ }^{6}$ Although this complication could be avoided using homoalkyl species, it is known that these magnesiates, such as $\left[\mathrm{LiMgBu}_{3}\right]$, have limited metalating power, ${ }^{2,7}$ and appear better suited for other reactions such as $\mathrm{Mg}$-halogen exchange or nucleophilic alkylation. ${ }^{8}$ Breaking new ground, here we report the synthesis and structural authentication of a new mixed potassium-alkylmagnesium reagent family. Assessing their reactivity with a range of arenes and heteroarenes, we present a new synthetically useful protocol to promote regioselective magnesiation under mild reaction conditions.
Potassium magnesiate $\left[\left(\mathrm{C}_{6} \mathrm{H}_{6}\right) \mathrm{KMgR}_{3}\right]$ (1) $\left(\mathrm{R}=\mathrm{CH}_{2} \mathrm{SiMe}_{3}\right)$ was prepared via co-complexation ${ }^{9}$ by mixing equimolar amounts of the monometallic alkyls $\mathrm{KR}$ and $\mathrm{MgR}_{2}$ (Scheme 1 and ESI). Using the monosilyl group is pivotal here as it offers enhanced solubility and stability compared to other C-based alkyl groups. ${ }^{10}$ Furthermore its large steric bulk greatly limits its nucleophilic power. Crystalline $\mathbf{1}$ was characterised by ${ }^{1} \mathrm{H}$ and ${ }^{13} \mathrm{C}$ NMR and structurally defined by X-ray crystallography (Fig. 1a, b). ${ }^{11}$

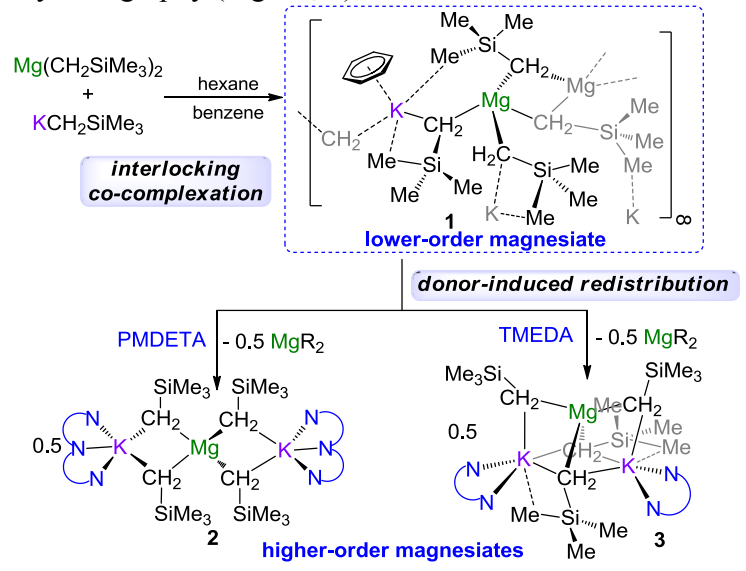

Scheme 1 Synthesis of potassium magnesiate 1 and donor-induced redistribution processes to give higher-order species $\mathbf{2}$ and $\mathbf{3}$.

$\mathrm{X}$-ray studies revealed a novel infinitely aggregated structure for $\mathbf{1}$ (Figure S1), ${ }^{11}$ combining $\mathrm{K}-\mathrm{C}$ and $\mathrm{Mg}-\mathrm{C}$ bonds with medium-long $\mathrm{K} \cdots \mathrm{Me}$ electrostatic interactions [ranging from 3.1352(18) to $3.4383(17) \AA]{ }^{12}$ Its basic repeat unit comprises a trigonal planar $\mathrm{Mg}$ bonded to three monosilyl groups (Figure 1a). Metals are connected by an alkyl group, which coordinates to $\mathrm{K}$ in an $\eta^{2}$-fasion through its $\mathrm{CH}_{2}$ unit and to one $\mathrm{SiMe}_{3} \mathrm{Me}$ group. $\mathrm{K}$ attains further coordination by $\pi$-engaging with a molecule of benzene and forming a long contact with a Me of another $\mathrm{R}$ group. One remaining alkyl binds in a similar ambidentate mode to a neighbouring $\mathrm{K}$ atom along the crystallographic $b$-axis; whereas the other $\mathrm{R}$ group interacts through its methylene head with a $\mathrm{Mg}$ atom located in a perpendicular disposition and through one of its Me groups with another $\mathrm{K}$ centre, giving rise to an intricate 2D network (Figure S1 and ESI for 

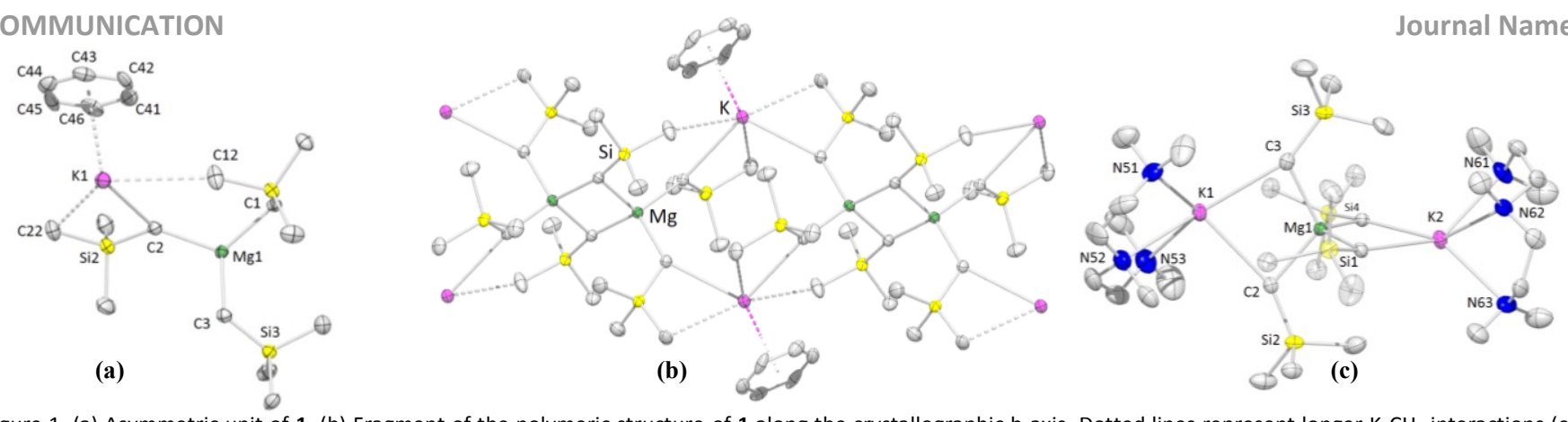

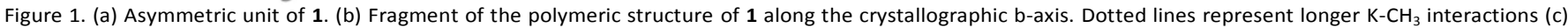
Molecular structure of $\mathbf{2}$ (displacement ellipsoids are drawn at the $50 \%$ probability level. $\mathrm{H}$ atoms and minor disorder components are omitted for clarity).

selected bond distances). ${ }^{11}$ Compound 1 shows excellent solubility in $\mathrm{C}_{6} \mathrm{D}_{6}$. Its ${ }^{1} \mathrm{H}$ and ${ }^{13} \mathrm{C}$ spectra revealed a single set of alkyl signals, ${ }^{11}$ suggesting that its polymeric constitution is not retained in solution. DOSY ${ }^{1} \mathrm{H}$ NMR studies, using internal standards, offer further support for the deaggregation of $\mathbf{1}$ in $\mathrm{C}_{6} \mathrm{D}_{6}$ solutions, estimating its size (in terms of FW) to be $433 \mathrm{~g} \mathrm{~mol}^{-1}$, consistent with monomeric $\left\{\mathrm{KMgR}_{3}\right\}$ units partially solvated by 1 or 2 molecules of $\mathrm{C}_{6} \mathrm{D}_{6}$ (Figures S5 and S6). ${ }^{11}$

Adding polydentate $\mathrm{N}$-donors PMDETA $\left(N, N, N^{\prime}, N^{\prime}, N^{\prime}\right.$ pentamethyldiethylenetriamine) or TMEDA $\left(N, N, N^{\prime}, N^{\prime}-\right.$ tetramethylethylenediamine) to a suspension of $\mathbf{1}$ in hexane, not only causes the deaggregation of its polymeric structure but also induces a redistribution process forming higher-order potassium rich magnesiates [(PMDETA $)_{2} \mathrm{~K}_{2} \mathrm{MgR}_{4}$ ] (2) and [(TMEDA) $\left.)_{2} \mathrm{~K}_{2} \mathrm{MgR}_{4}\right]$ (3) with the concomitant elimination of $\mathrm{MgR}_{2}$ (Scheme 1). ${ }^{13}$

$\mathrm{X}$-ray studies revealed trinuclear structures for $\mathbf{2}$ and $\mathbf{3}^{14}$ (Figure 1c and S3 respectively). ${ }^{11}$ Both compounds display a central C4coordinated $\mathrm{Mg}$ atom flanked by two donor-solvated $\mathrm{K}$ cations. Interestingly, the different hapticities of the N-ligands impose remarkable differences in their structural motifs. Thus, containing PMDETA ligated 2, displays a linear $\mathrm{K}^{\cdots} \mathrm{Mg} \cdots \mathrm{K}$ arrangement $\left[174.18(3)^{\circ}\right]$ with each $\mathrm{R}$ group bridging $\mathrm{Mg}$ with a $\mathrm{K}$ centre (Fig. 1c). ${ }^{13}$ Contrastingly, in TMEDA-solvate $\mathbf{3}$, in order to attain coordinative saturation, each $\mathrm{K}$ needs to bind to three monosilyl groups. Thus two of the four $\mathrm{R}$ groups in $\mathbf{3}$ bridge between the three metals (through $\mathrm{C} 8$ and $\mathrm{C} 5$ in Fig. S2) through their $\mathrm{CH}_{2}$ heads, while the remaining ones connect $\mathrm{Mg}$ with only one $\mathrm{K}$ atom. These distinct bonding modes must contribute significantly to the marked non-linearity of the $\mathrm{K}^{\cdots} \mathrm{Mg}{ }^{\cdots} \mathrm{K}$ vector $\left[100.14(19)^{\circ}\right]$. Unlike 2, in 3 there are several $\mathrm{K} \cdots \mathrm{Me}$ medium-long contacts, one of which involves a $\mathrm{K}$ atom from a neighboring unit, giving rise to an extended linear chain structure (Fig. S4). ${ }^{11}$ Structurally defined homoalkyl potassium magnesiates are extremely scarce, with the only precedents being allyl polymers $\left[\mathrm{KMg}\left(\mathrm{C}_{3} \mathrm{H}_{5}\right)_{3}(\mathrm{THF})_{2}\right]$ and $\left[\mathrm{K}_{2} \mathrm{Mg}\left(\mathrm{C}_{3} \mathrm{H}_{5}\right)_{4}(\mathrm{THF})\right]$. ${ }^{15}$

${ }^{1} \mathrm{H}$ and ${ }^{13} \mathrm{C}$ NMR analysis of 2 and $\mathbf{3}$ suggests that the solid-state monomeric contacted ion pair $\left[(\mathrm{N} \text {-donor })_{2} \mathrm{~K}_{2} \mathrm{MgR}_{4}\right]$ constitutions observed in the solid state are retained in $\mathrm{C}_{6} \mathrm{D}_{6}$ solution. ${ }^{11}$

To probe the metallating abilitiy of potassium magnesiates $\mathbf{1 - 3}$, we chose anisole (4a) as a substrate, a benchmark molecule in directed ortho metallation (DoM) (Table 1). ${ }^{16}$

Control reactions of equimolar amounts of the homometallic components of 1-3, KR and $\mathrm{MgR}_{2}$ with $4 \mathrm{a}$ in hexane at $20^{\circ} \mathrm{C}$ for $2 \mathrm{~h}$ showed sluggish reactivity. Whereas the addition of PMDETA does not seem to affect the reactivity of $\mathrm{MgR}_{2}$, it does increase the performance of KR (Table 1, entries 1-2). However, the resulting aryl potassium intermediate is unstable and slowly decomposes. Probing the reactivity of the new potassium magnesiates, using lower-order 1 led to similar low conversions as those observed with KR (Table 1, entry 3). Remarkably, higher-order magnesiate 2 proved an excellent magnesiating reagent, affording conversions of
$99 \%$ (Table 1, entry 4). Subsequent trapping of the metallated intermediate 5a with iodine furnished 2-iodoanisole (6a) in near quantitative isolated yield (92\%). Similarly, 5a undergoes carboxylation with $\mathrm{CO}_{2}$, yielding 2-methoxybenzoic acid (6a') in $84 \%$ yield.

Table 1. Metalation of anisole (4a) using different alkyl Li, $\mathrm{Na}, \mathrm{K}$ and $\mathrm{Mg}$ reagents.

\begin{tabular}{|c|c|c|}
\hline $\mathrm{OMe}$ & \multicolumn{2}{|l|}{$\mathrm{OMe}$} \\
\hline & $\mathrm{I}_{2}$ (excess) & $\begin{array}{l}\mathrm{MR}=\mathbf{2} \text { (1 equiv) } \\
\mathbf{6 a}: 92 \% \mathrm{x}=1\end{array}$ \\
\hline & or $\mathrm{CO}_{2}$ (excess) & $6 a^{\prime}: 84 \% \hat{x}=\mathrm{COOH}$ \\
\hline $4 a$ & $2 \mathrm{n},-\mathrm{sivie}_{4}$ & \\
\hline Entry & $\mathbf{M R}^{a}$ & $\begin{array}{l}\text { Conversion } \\
\text { to } \mathbf{5 a}(\%)^{b}\end{array}$ \\
\hline 1 & $\mathrm{MgR}_{2}$ & $0(0)^{c}$ \\
\hline 2 & $\mathrm{KR}$ & $20(57)^{c}$ \\
\hline 3 & $\mathrm{KMgR}_{3}(\mathbf{1})$ & 15 \\
\hline 4 & $(\mathrm{PMDETA})_{2} \mathrm{~K}_{2} \mathrm{MgR}_{4}$ (2) & 99 \\
\hline 5 & $(\mathrm{TMEDA})_{2} \mathrm{Li}_{2} \mathrm{MgR}_{4}$ & 5 \\
\hline 6 & $(\mathrm{TMEDA})_{2} \mathrm{Na}_{2} \mathrm{MgR}_{4}$ & 10 \\
\hline 7 & $\left(\mathrm{PMDETA}_{2} \mathrm{~K}_{2} \mathrm{MgR}_{4}(3)+18\right.$-crown-6 & 0 \\
\hline
\end{tabular}

${ }^{a} 1.0 \mathrm{mmol}$ of $4 \mathbf{a}$ and $1.0 \mathrm{mmol}$ of $\mathbf{M R}$ were used. ${ }^{b}$ Conversion was determined by $\mathrm{GC}$ analysis of iodolysed reaction aliquots, using an internal standard (heptadecane). ${ }^{c}$ In brackets conversion shown when adding 0.5 mmol of PMDETA.

Disclosing a dramatic alkali-metal effect, attempts to achieve an efficient metallation of 4a using related higher-order lithium- and sodium magnesiates [(TMEDA $)_{2} \mathrm{Li}_{2} \mathrm{MgR}_{4}$ ] (which displays the same structure as 2) ${ }^{18}$ and [(TMEDA) ${ }_{2} \mathrm{Na}_{2} \mathrm{MgR}_{4}$ ] were unsuccessful (Table 1, Entries 5-6). Further evidence of the crucial role of $\mathrm{K}$ for the success of these magnesiation reactions was found when the reaction of 2 with $4 \mathbf{a}$ was carried out in the presence of 2 equivalents of 18crown-6, which is known to have a high affinity towards potassium cations, which completely shuts down metallation (Table 1, entry 7). These findings suggest that these reactions must operate via contacted-ion pair mixed-metal intermediates (CIP), being genuine examples of potassium-mediated magnesiations. The enhanced reactivity of $\mathbf{2}$ can be rationalised by the synergic partnership between the metals. By forming a higher-order magnesiate, the low kinetic basicity of the $\mathrm{Mg}-\mathrm{C}$ bonds in $\mathrm{MgR}_{2}$ can be overcome, but the $\mathrm{K}$ cations also play a major role in the process, coordinating to anisole, bringing its ortho-H in close proximity to the activated magnesiate anion. Interestingly, studies using sub-stoichiometric amounts of 2, demonstrate its ability to display polybasic behaviour (Table S1, ESI). Using 0.33 and 0.25 equivalents of 2 leads to 88 and $74 \%$ conversions of 4 a respectively, which suggest that up to three of its four potential basic arms are active in the deprotonation reaction. ${ }^{11}$

In order to explore the scope of $\mathbf{2}$, we next studied its reactivity towards several unsaturated substrates (Table 2). 
Table 2. Potassium-mediated-magnesiations of various aromatic and heteroaromatic substrates using 2

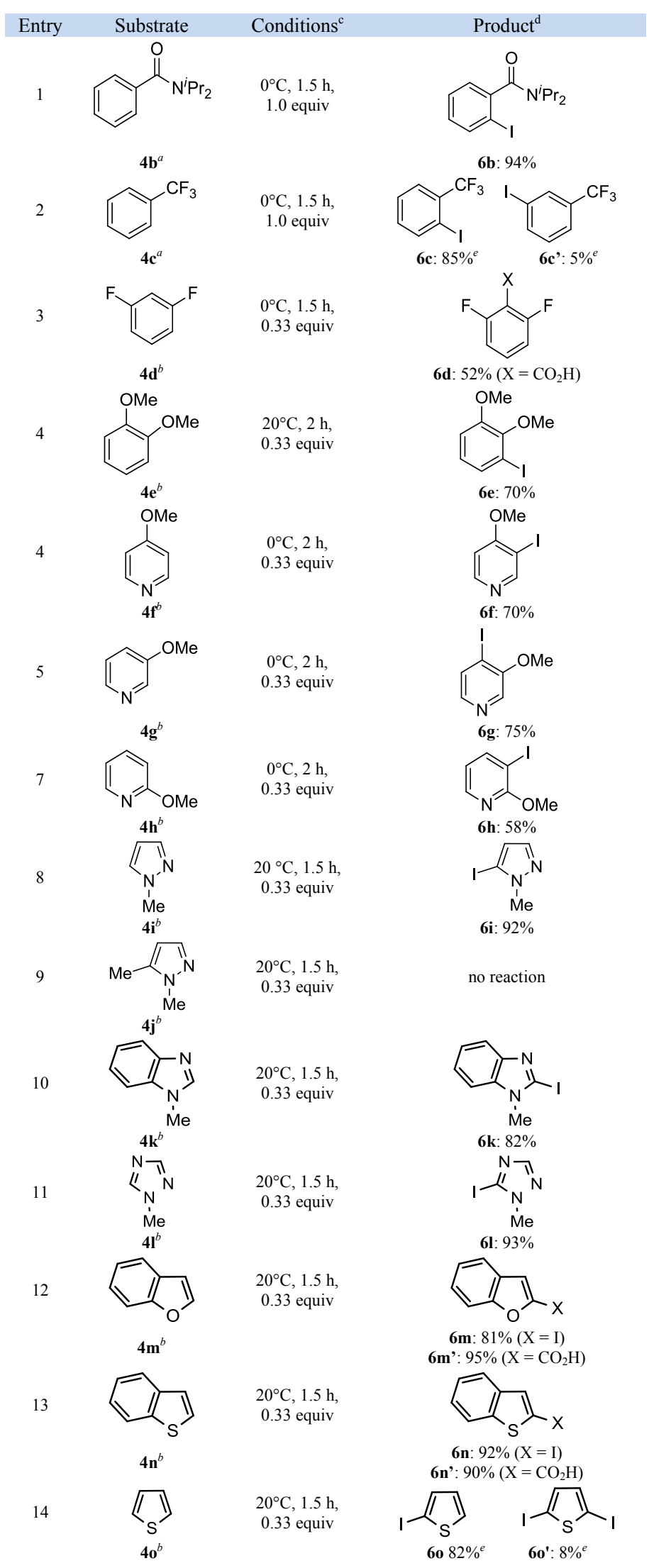

${ }^{a} 0.5 \mathrm{mmol}$ of substrate was used. ${ }^{b} 1.5 \mathrm{mmol}$ of substrate were used. ${ }^{c}$ Time, temperature and amount of base 2 for the metalation reactions. ${ }^{d}$ Yields refer to isolated yields of analytically pure products $(>95 \%$ purity determined by NMR or GC-analysis), unless otherwise stated. ${ }^{e}$ Yield was determined by ${ }^{1} \mathrm{H}-$ NMR analysis using an internal standard (ferrocene).

The amido group $\left(-\mathrm{CONR}_{2}\right)$ is regarded a strong ortho-directing group, and metalation using 2 proceeded smoothly and efficiently at $0^{\circ} \mathrm{C}$, leading to 2-iodo- $N, N$-diisopropylbenzamide $(\mathbf{6 b})$ in $94 \%$ yield, following $\mathrm{I}_{2}$ quenching (Table 2, Entry 2). Metallation of trifluoromethylbenzene $\mathbf{( 4 c )}$ formed two ortho and meta metallated regioisomers in 85 and 5\% yields (Table 2, Entry 1). Reaction of 2 with 1,3-difluorobenzene (4d) occurred at the most acidic 2-position with carboxylation furnishing the tri-substituted arene 6d in $52 \%$ yield (Table 2, Entry 3 ). Similarly 1,2-dimethoxybenzene $4 \mathbf{e}$ reacts with 0.33 eq of $\mathbf{2}$ at room temperature to exclusively afford $\mathbf{6 e}$ in a $70 \%$ yield (Table 2, Entry 4). Due to their vast synthetic relevance, selective functionalization of pyridines using metallation under mild conditions is highly desirable. Generally, organolithium metalation reactions need to be carried out at $-78^{\circ} \mathrm{C}$ and regioselective control can be challenging. We tested the reaction of $\mathbf{2}$ with the three different regioisomers of 2-, 3- and 4-methoxypyridine (4e-g, Table 2, Entries 4-6). Although ether-functionalities are not regarded as strong directing groups, ${ }^{16 a}$ deprotonation occurred in all three cases regioselectively ortho to the OMe group under mild conditions $\left(0{ }^{\circ} \mathrm{C}\right.$, $2 \mathrm{~h}$ ), producing iodopyridines $6 \mathrm{e}-\mathrm{g}$ in $58-75 \%$ isolated yield. ${ }^{19}$

Moving on to diazoles, the metallation of 1-methyl-1H-pyrazole (4i) proceeded efficiently in the 5-position and iodination furnished $\mathbf{6 i}$ in near quantitative yield using 0.33 equivalents of 2 (92\%, Table 2, Entry 8). However, on blocking the 5-position by a Me substituent (4j), no metallation was observed (Table 2, Entry 9). The similar heterocycles 1-methyl-1H-benzo[d]imidazole (4k) and triazole derivative $4 \mathbf{l}$ were efficiently metallated and iodinated at room temperature, affording $\mathbf{6 k}$ and $\mathbf{6 l}$ in excellent yields $(82-93 \%$; Table 2, Entries 10-11). Finally, oxygen and sulphur containing heterocycles $(\mathbf{4 m - 0})$ did not pose any problems and reaction with 2 led to regioselective deprotonation at the 2-position. The corresponding iodinated (6m-0) and carboxylated (6n', 40') heterocycles were produced in equally high yields $(81-95 \%$, Table 2 , Entries 12-14). With unsubstituted thiophene (40) $8 \%$ of twofold metallation leading to 2,5-diiodothiophene (4o') was observed.

In summary, we have uncovered the structures of a new family of homoalkyl potassium magnesiates. Revealing an important donor effect, addition of polydentate N-Lewis bases to the highly aggregated magnesiate $\mathbf{1}$, not only induces formation of smaller aggregates but also changes the mixed-metal formulation, to higherorder magnesiates 2 and 3. A new straightforward methodology, using 2 as a metallating reagent, to promote regioselective $\mathrm{Mg}-\mathrm{H}$ exchange of a wide range of aromatic and heteroaromatic substrates under mild reaction conditions has been disclosed. Our studies suggest the involvement of CIP intermediates, with the presence of $\mathrm{K}$ being the key to facilitate these novel magnesiation reactions.

We thank the EPSRC, the Carnegie Trust for the Universities of Scotland the Royal Society and the ERC for their generous sponsorship of this research and Professor R. E. Mulvey for insightful discussions.

\section{Notes and references}

${ }^{a}$ WestCHEM, Department of Pure and Applied Chemistry, University of Strathclyde, Glasgow, G1 1XL.UK.eva.hevia@strath.ac.uk.

${ }^{b}$ School of Chemistry, Newcastle University, Newcastle upon Tyne NE1 $7 R U, U K$.

Electronic Supplementary Information (ESI) available: [full experimental details, X-ray crystallographic data and copies of the NMR spectra]. See DOI: $10.1039 / \mathrm{c} 000000 \mathrm{x} /$ 
1 (a) M. Schlosser in Organometallics in Synthesis. A Manual, $2^{\text {nd }}$ ed. (Ed. M. Schlosser), Wiley, Chichester, 2002, p 1. (b) E. Carl and D. Stalke in Lithium Compounds in Organic Synthesis: From Fundamentals to Applications, ed. R. Luisi and V. Capriati, WileyVCH Verlag \& Co, Weinheim, Germany, 2014.

2 F. Mongin, A. Harrison-Marchand, Chem. Soc. Rev. 2013, 113, 7563.

3 R. E. Mulvey, F. Mongin, M. Uchiyama, Y. Kondo, Angew. Chem. Int. Ed. 2007, 46, 3802. (b) B. Haag, M. Mosrin, H. Ila, V. Malakhov, P. Knochel, Angew. Chem. Int. Ed. 2011, 50, 9794.

4 A. J. Martinez, D. R. Armstrong, b. Conway, B. J. Fleming, J. Klett, A. R. Kennedy, R. E. Mulvey, S. D. Robertson, C. T. O'Hara, Chem. Sci. 2014, 5, 771 .

5. C. Unkelbach, D. F. O'Shea, C. Strohmann, Angew. Chem. Int. Ed. 2014, 53, 553.

6 W. Clegg, B. Conway, P. Garcia-Alvarez, A. R. Kennedy, R. E. Mulvey, L. Russo, J. Sasmannshausen, T. Tuttle, Chem. Eur. J. 2009, 15, 10702 .

7 H. Haward, O. Bayh, C. Hoarau, F. Trécourt, G. Quéguiner, F Marsais, Tetrahedron, 2008, 64, 3236.

8 M. Hatano, T. Matsumura, T. Ishihara, Org. Lett. 2005, 7, 573.

9 S. E. Baillie, W. Clegg, P. Garcia-Alvarez, E. Hevia, A. R. Kennedy, J. Klett, L. Russo, Chem. Commun. 2011, 47, 388.

10 W. Clegg, B. Conway, A. R. Kennedy, J. Klett, R. E. Mulvey, L. Russo, Eur. J. Inorg. Chem. 2011, 721.

11 See ESI for details.

12 These values are similar to those previously reported for the structure of $[\mathrm{MeK}]$ where $\mathrm{K}$ also forms long distance interactions with the $\mathrm{Me}$ groups of other units (mean K...Me, $3.441 \AA$ ) which are significantly elongated when compared to the $\mathrm{K}-\mathrm{Me}$ distances towards the carboanion (2.447 $\AA$ ), see E. Weiss, T. Lambertsen, B. Schubert, J. K. Cockcroft, J. Organomet. Chem. 1988, 358, 1 .

13 Compounds 2 and $\mathbf{3}$ can also be rationally synthesized by reaction of the relevant $\mathrm{N}$-donor, $\mathrm{KR}$ and $\mathrm{MgR}_{2}$ in a 2:2:1 ratio (isolated crystalline yields 78 and $45 \%$ for 2 and $\mathbf{3}$ respectively).

14 Similar linear arrangements have been described as classical "Weiss motifs" in related $\mathrm{Li}$ and $\mathrm{Na}$ magnesiate structures, see: E. Weiss, Angew. Chem. Int. Ed. 1993, 32, 1501.

15 C. Lichtenberg, T. P. Spaniol, I. Peckermann, T. P. Hanusa, J. Okuda, J. Am. Chem. Soc. 2013, 135, 811 .

16 For DoM reactions see: a) V. Snieckus, Chem. Rev. 1990, 90, 879; b) A. Wagner, J. R. Desmurs, V. Pe, C. Mioskowski, Angew. Chem. Int. Ed. Engl. 2002, 41, 340; c) S. Wunderlich, T. Bresser, C. Dunst, G. Monzon, P. Knochel, Synthesis 2010, 2670.

17 For an insightful discussion on the ortho-lithiation of anisole see reference $16 \mathrm{a}$.

18 S. E. Baillie, W. Clegg, P. Garcia-Alvarez, E. Hevia, A. R. Kennedy, J. Klett, L. Russo, Organometallics, 2012, 31, 5131.

19 For regioselective metalation of $4 \mathrm{e}$ in 2-position and $\mathbf{4 g}$ in 6-position overriding the directing effect see: a) S. M. Manolikakes, N. M. Barl, C. Sämann, P. Knochel, Z. Naturforsch. 2013, 68b, 411; b) M. Jaric, B. A. Haag, A. Unsinn, K. Karaghiosoff, P. Knochel, Angew. Chem. Int. Ed. 2010, 49, 5451. 